\title{
LE MAPPE DI SALUTE DI UNA POPOLAZIONE STORICA PRIME INDAGINI SUL FRIULI (XIX SECOLO)
}

\author{
HEALTH MAPS OF A HISTORICAL \\ POPULATION IN NINETEENTH-CENTURY \\ FRIULI (NORTHEASTERN ITALY)
}

\author{
Alessio Fornasin", Marco Breschi", Matteo Manfredini" ${ }^{* * *}$
}

\begin{abstract}
SuMMARY
This paper aims at presenting some health maps of a historical population. The studies on the health status of past populations are usually focused on the causes of death. Our purpose is to present some descriptive analyses on non-deadly diseases. The present work focuses on the province of Friuli (north-eastern Italy) in the second half of the nineteenth century. The used sources are military call-up records. We collected about 300,000 records relative to military recruitment that took place between 1866 and 1909 (birth cohorts I846-I890). Our main concern was the health status of the 20-year male population, and its association with environmental, socio economic and genetic factors. Generally speaking, we observe that the northern mountain area was the most advantaged, while young adults from the eastern and western parts of the province were the most disadvantaged. A lot of factors and causes contributed to determine the spatial distribution of specific diseases in Friuli, whilst others remain unknown because simple descriptive analyses are not sufficient to highlight them all. In particular, the distribution of the thyroidal hypertrophy was almost certainly due to the water quality, while the distribution of dental caries was probably related to genetic and dietary factors.
\end{abstract}

Key words: Health maps; Military sources; Friuli (Italy); $19^{\text {th }}$ century.

* Dipartimento di Scienze economiche e statistiche - Università di Udine, Udine, Italia.

** Dipartimento di Scienze economiche e aziendali - Università di Sassari, Udine, Italia.

*** Dipartimento di Bioscienze - Università di Parma, Parma, Italia.

Corrispondenza: Alessio Fornasin, Università degli Studi di Udine, Dipartimento di Scienze Economiche e Statistiche, Via Tomadini, 30/A - I-33100 Udine, Italia.

E-mail: fornasin@uniud.it. 


\section{INTRODUZIONE}

Lo stato di salute di una popolazione è una delle componenti più importanti del suo benessere. Una popolazione più sana vive più a lungo e può meglio dispiegare le sue capacità produttive e creative. Alti standard di benessere diffusi su tutta la popolazione sono lo specchio della civiltà e della ricchezza di un paese, la cui storia può essere a sua volta ricostruita seguendo l'evoluzione e i miglioramenti della salute dei suoi abitanti.

Dal punto di vista storico, le ricerche sulla salute delle popolazioni hanno messo in rilievo il passaggio da un modello epidemiologico dominato dalla mortalità ad un modello epidemiologico dominato dalla morbilità (Bernabeu Mestre 1995). Le grandi trasformazioni in campo medico e sanitario hanno anche segnato il passaggio dalla predominanza delle cause di morte dovute alle malattie trasmissibili a quelle non trasmissibili. Questo processo è stato denominato transizione epidemiologica. Le ricerche sul tema si sono orientate lungo due linee. La prima, che ha posto l'attenzione sul punto di vista demografico, ha privilegiato gli studi sulla mortalità e sulle cause di morte (Sori I984). In questi casi, oltre agli studi sulla mortalità generale o, spesso, sulla mortalità infantile e nei primi anni di vita (Pozzi 2000; Breschi, Fornasin 2007), hanno trovato largo spazio le indagini su singoli episodi epidemici: peste, colera, influenza (Alfani 20I0, Tognotti 2000; 2002); o su malattie endemiche: malaria, pellagra (Snowden 2006, Livi Bacci 1986). La seconda ha indagato, da una parte, i progressi della medicina e l'evoluzione dell'organizzazione sanitaria e dei sistemi di welfare, e dall'altra l'evoluzione della legislazione sui temi dell'igiene e della sanità pubbliche (Donelli, Di Carlo 2002; Giovannini 1996). Questi aspetti hanno ricevuto particolare attenzione con riferimento allo sviluppo industriale, quando hanno cominciato a generarsi nuovi bisogni trainati dal mutare delle condizioni sociali oltre che lavorative (Betri, Gigli Marchetti 1982).

Sebbene nel tempo l'attenzione degli studiosi si sia spostata da un ambito pretransizionale ad uno posttransizionale, molti aspetti che sono oggi oggetto di studio non mancavano di sussistere anche nel passato, benché fossero molto meno evidenti rispetto a quelli tradizionalmente indagati. Ad esempio non esistono praticamente studi sugli incidenti sul lavoro prima della nascita dell'industria e della legislazione sul welfare, o sulle disabilità prima di quella che è forse la più efficiente fabbrica di disabilità della storia, ovvero la Prima guerra mondiale. Tuttavia gli incidenti sul lavoro si verificavano anche al di fuori delle fabbriche e le ferite non erano provocate solo dall'artiglieria sui 
campi di battaglia, con la triste generazione di invalidi e mutilati, così come vi erano patologie oggi facilmente curabili che però, fino ad un passato non molto lontano, pregiudicavano in maniera irrimediabile la salute e in qualche caso anche la vita delle persone: una semplice frattura, la cataratta, una piccola ernia...

Così poca attenzione su questi aspetti è in parte dovuta all'assenza di fonti o al loro ridotto potere informativo. Parziale eccezione a questa generale mancanza di lavori sull'argomento è data dagli studi condotti sulle fonti di carattere militare (Farolfi I982). Questi approfondimenti sono però possibili solo relativamente a quei paesi, come l'Italia, dove la leva militare era universale e quindi la relativa visita medica era obbligatoria per tutti. Pur se limitato ad un numero circoscritto di contesti territoriali, lo spoglio sistematico delle visite di leva ha già permesso, in diversi casi, di esplorare alcuni aspetti dello stato di salute della popolazione (Bussini, Lanari 2007; Bussini et al. 2008; Cau et al. 2007; Gatti et al. 2008). Naturalmente il limite di tutte queste analisi è costituito dal fatto che le visite, anche là dove sono universali, riguardano solamente un segmento ristretto della popolazione, vale a dire i giovani maschi di una determinata generazione. Il limite è insuperabile, ma certo la fonte permette di gettare uno sguardo sulle condizioni di salute più generali della popolazione. Del resto le visite di leva rappresentano la prima raccolta sistematica di dati sullo stato di salute. Infatti, gli studi di sanità militare, già nella seconda metà dell'Ottocento, erano quasi diventati un campo di ricerca a sé, alimentato proprio dalla grande mole di informazioni disponibili, e che trattava un ampio ventaglio di temi (Sormani r88I, I3-6I; Quinzio I888).

Oggi la costruzione di mappe della salute è resa possibile dalla raccolta sistematica di informazioni di carattere medico e sanitario e svolge un ruolo di grande importanza nell'individuare le origini delle singole malattie e anche nel determinarne la causa. Disegnare mappe di questo tipo era assai più difficile nel passato, ma quando sono state realizzate hanno dato risultati di grande interesse, che ancora oggi sono la base per molte ricerche di carattere storico. Alcuni dei primi lavori effettuati con riguardo all'Italia, dovuti a Ridolfo Livi (I896), sono stati creati proprio a partire dalle fonti militari.

$\mathrm{Ci}$ sono naturalmente altre raccolte di informazioni che permettono di descrivere fenomeni legati alla salute della popolazione e che hanno dato anche luogo alla creazione di mappe tematiche. Tra queste ci sono le carte nosologiche della malaria, del tracoma, del vaiolo (Torelli I882; Alvaro 1903). In altri casi ad essere rilevati sono i decessi causati da singole grandi epidemie. 
Del resto è proprio riguardo al colera che fu effettuato il primo riuscito tentativo di illustrare la diffusione di un fenomeno epidemico, ovvero la celebre mappa di Londra di John Snow (1855). Altre informazioni furono raccolte in occasione dei primi censimenti del Regno d'Italia, ma la qualità di questi dati era assai limitata rispetto a quella delle fanti di origine militare. Fino al censimento del ıوı erano posti dei quesiti relativi alle infermità, come la cecità, il sordomutismo e malattie mentali (Mastroluca, Verrascina 2012, 99).

La raccolta di informazioni a livello territoriale effettuata nel passato permette oggi con una certa facilità di costruire mappe storiche della salute, operazione enormemente facilitata dalla diffusione delle tecnologie Gis. Ciò nonostante i tentativi di questo tipo relativamente al nostro paese non sono numerosi (ad esempio Fornasin et al. 20II).

In questo lavoro intendiamo sondare le possibilità di una fonte: i registri della leva militare. L'esito delle nostre esplorazioni è condensato in una serie di mappe che rivelano la distribuzione di alcune caratteristiche dei giovani sottoposti alla visita di leva che più o meno direttamente possono essere collegate alla loro salute. L'analisi verte in particolare su alcune caratteristiche dello stato di salute o di benessere che hanno una chiara distribuzione territoriale, nell'intento di individuarne alcune possibili cause. Un altro obiettivo di questo lavoro è quello di off rire alcuni elementi di carattere metodologico che permettano di scavare in questo argomento. L'ampia messe di dati tratti dalle visite di leva non è qui ancora sistematizzata, tuttavia l'articolo intende rappresentare un primo passo verso la costruzione di mappe di salute di una popolazione storica e la loro interpretazione.

\section{IL TERRITORIO}

Il territorio di riferimento per questa indagine è la Provincia del Friuli, posta all'estremo nord est del Regno d'Italia. Dal punto di vista ambientale, percorrendo il territorio da sud verso nord, si incontrano molte situazioni diverse: la bassa pianura umida, composta da terreni prevalentemente argillosi e innervata da numerosi corsi d'acqua; l'alta pianura, costituita da terreni altamente permeabili e povera di acqua superficiale; la collina, particolarmente estesa nell'area a nord ovest del capoluogo Udine e, infine, la montagna che copre quasi la metà della superficie complessiva della provincia.

Dal punto di vista demografico, il Friuli contava nel I88I 528.559 residenti. La popolazione era distribuita in maniera diseguale sul territorio seguendo, nella sostanza, le differenti condizioni ambientali (Fig. I). Si addensava 


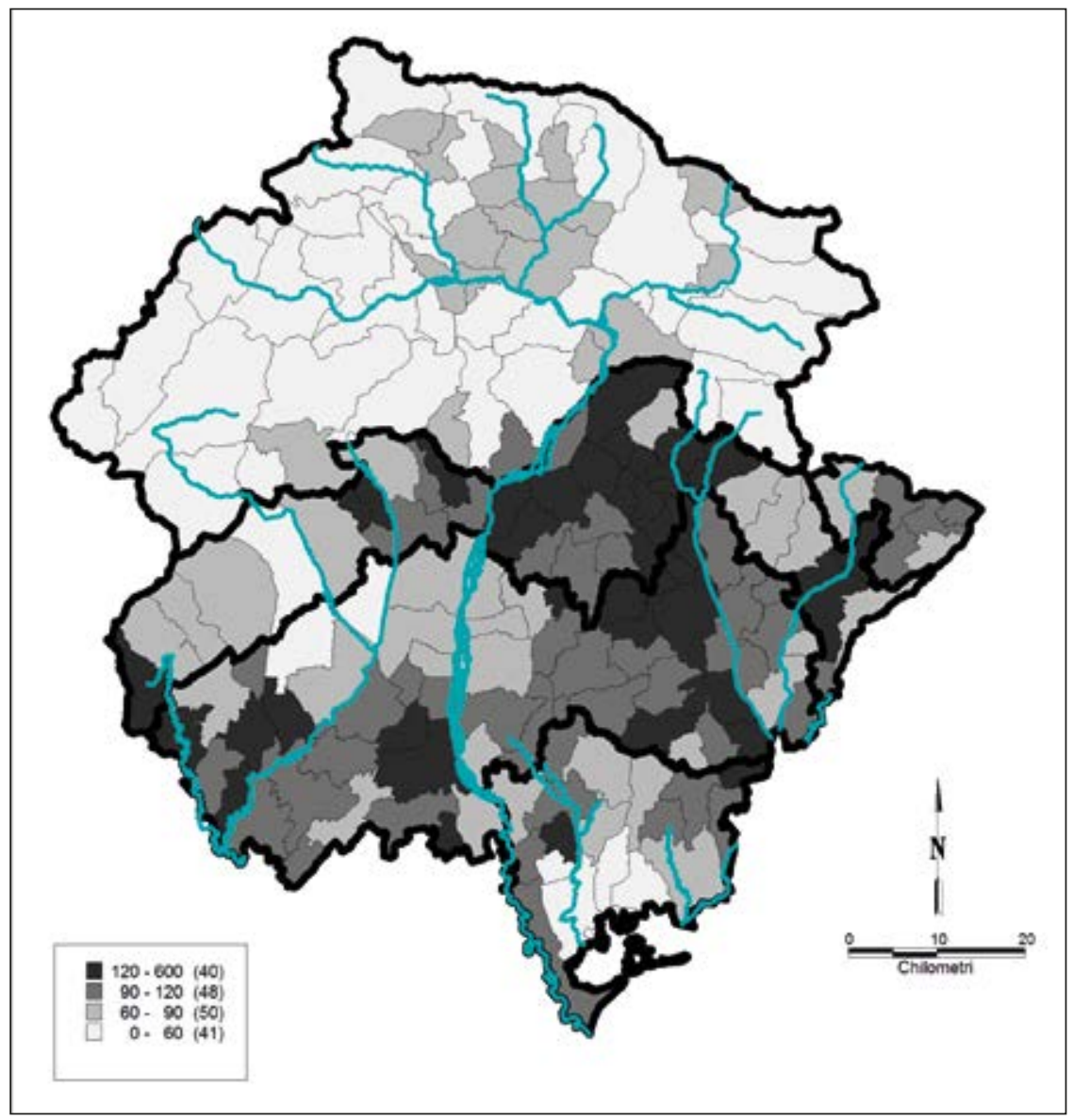

Fig. I. Densità della popolazione I88I (abitanti/km2). Fonte: Maic 1883.

in particolare nell'area collinare attorno al capoluogo Udine e nell'area sud orientale. La parte montana, e in particolare quella settentrionale, era la meno abitata, ma anche la bassa pianura meridionale, a causa dell'elevata insalubrità delle terre prossime al mare, era caratterizzata da una bassa densità di popolazione.

Ancora nel corso dell'Ottocento, le differenze di carattere geografico condizionavano pesantemente le caratteristiche economiche dei singoli territori. L'agricoltura era l'attività di gran lunga prevalente tra la popolazione della pianura, mentre assumeva importanza via via minore con l'aumentare dell'altitudine; a cambiare non era solo la proporzione degli occupati nel settore primario, ma anche le coltivazioni e il tipo di produzione. Nel piano era diffusa un'agricoltura di tipo promiscuo, parzialmente orientata al mercato, 
che si fondava da una parte sulle colture cerealicole, in cui dominava il binomio frumento-mais (ma si coltivavano, seppur in forma residuale, cereali minori, come il sorgo rosso e il grano saraceno), e dall'altra sulla vite e sul gelso. In montagna, invece, dove la produzione di grani era deficitaria ed era sufficiente a coprire il fabbisogno per soli due-tre mesi all'anno, prevaleva l'allevamento (Bianco 1994; Morassi 2002).

Per quel che concerne il quadro alimentare, la principale e quasi esclusiva fonte di nutrimento degli abitanti della pianura era costituita dai cereali, e in particolare dal mais. In ambito urbano si consumava molto frumento, anche se, bisogna ritenere, una non piccola parte della popolazione doveva alimentarsi a cereali primaverili. Anche la montagna, o almeno parte di essa, era caratterizzata da una maggiore varietà nei consumi rispetto alla pianura, grazie ad una produzione agricola più diversificata ed alla alternativa rappresentata dal patrimonio zootecnico e dalla produzione lattiero-casearia (Fornasin 2005a). L'autoconsumo era la norma nelle aree rurali, mentre l'area montana, per far fronte al suo fabbisogno alimentare, doveva importare cereali dalla pianura.

Un secondo elemento distintivo dell'economia friulana della seconda metà dell'Ottocento era dato dall'esistenza di importanti flussi migratori di carattere prevalentemente maschile e stagionale che si indirizzavano verso l'Europa centrale ed orientale. Benché, in termini generali, le occupazioni legate all'emigrazione fossero più o meno le stesse in tutto il territorio - i mestieri degli emigranti si esplicavano per la maggior parte nell'ambito dell'edilizia e delle costruzioni -, al nord, nella parte montana, gli emigranti erano di solito più numerosi che nel sud. La differenza nasceva anche dal fatto che le tradizioni migratorie erano alquanto diverse a seconda del contesto territoriale: in montagna l'emigrazione stagionale era una realtà consolidata da secoli, sebbene fino alla prima metà dell'Ottocento si basasse su attività artigianali o commerciali (Fornasin, I998); nel piano, invece, quantunque non fosse certo sconosciuta, divenne un fenomeno sociale ed economico di grande rilevanza solo nel corso del XIX secolo (Di Caporiacco r967-69).

\section{LA FONTE E I DATI}

La fonte utilizzata in questo lavoro è costituita dalle liste di estrazione militare, ovvero i registri che riportano l'esito delle visite mediche a cui erano sottoposti tutti i giovani che avevano raggiunto l'età per prestare servizio nelle forze armate. In Italia il servizio militare era universale, vale a dire 
che tutti i giovani di una determinata generazione dovevano presentarsi alla visita di leva. La visita serviva ad accertare chi fosse idoneo, cioè «abile» a prestare servizio nei ranghi dell'esercito. Non tutti gli idonei vestivano però la divisa. Infatti, fino al 1889 , il numero di giovani di una generazione che avrebbe svolto il servizio militare era stabilito ogni anno con una apposita legge, che suddivideva il contingente di chiamati sulla base dei mandamenti militari in cui era suddiviso l'intero paese.

Per poter garantire una equa ripartizione dei giovani chiamati al servizio tra i comuni che costituivano i singoli distretti militari, i giovani della classe di leva del distretto venivano estratti a sorte e i nominativi costituivano un unico elenco in cui, evidentemente, risultavano mescolati giovani di tutti i comuni. A questo punto, seguendo l'ordine dell'elenco, i giovani venivano visitati. Gli esiti possibili erano principalmente tre: abile alle armi, rivedibile e riformato. Tutti i giovani considerati abili venivano suddivisi in 3 classi. Nella prima erano elencati gli abili che, secondo l'ordine di estrazione, andavano ad esaurire il contingente previsto per il distretto. Nella seconda tutti i giovani nella medesima condizione che però eccedevano il contingente stabilito. Nella terza, invece, tutti coloro che, pur essendo risultati idonei dal punto di vista fisico, avevano diritto all'esenzione per ragioni familiari, ad esempio i figli di madre vedova o coloro che già avevano un fratello sotto le armi.

Coloro che non risultavano abili non necessariamente venivano riformati. Se la disabilità riscontrata in sede di visita non era giudicata grave o era comunque suscettibile di miglioramento, questi giovani erano inseriti nell'elenco dei rivedibili, ed erano rimandati alla leva successiva. In questo caso i loro nomi non rientravano più tra quelli estraibili a sorte, ma, sulla base dell'ordine con cui erano stati visitati andavano a costituire il gruppo dei primi ad essere sottoposti a nuova visita. I riformati, infine, erano esclusi dal prestare servizio nell'esercito.

Benché in termini generali la procedura seguita fosse quella qui brevemente descritta, nel tempo le regole, o anche solo la prassi, avevano conosciuto alcuni aggiustamenti. Nei primi anni della leva obbligatoria del Regno d'Italia, ad esempio, coloro che avevano diritto all'esenzione non erano visitati, quindi non disponiamo delle informazioni relative ad un numero rilevante di giovani.

Come abbiamo visto, nella seconda metà dell'Ottocento, anche il Friuli conobbe una crescita dei flussi migratori verso l'estero. Nella documentazione 
militare i riflessi dell'emigrazione sono consistenti. Sempre più spesso, infatti, nei registri viene riportata l'informazione di giovani visitati all'estero o che comunque sono messi a ruolo in via provvisoria senza visita o, almeno, senza una visita che abbia lasciato traccia documentaria nelle liste di estrazione. La dicitura utilizzata per designare l'esito della visita di questi giovani è «arruolato» $\mathrm{o}$ «arruolato per procura». Oltre agli «arruolati», sempre più spesso, mano a mano che ci inoltriamo nell'Ottocento troviamo giovani «renitenti», persone cioè che non si erano presentate alla visita senza addurre giustificazione alcuna. Molti di questi renitenti sono in realtà giovani emigrati all'estero, in molti casi anche persone che oramai risiedevano stabilmente fuori dall'Italia. Anche per i renitenti, naturalmente, così come per molti degli «arruolati» con visita all'estero mancano le informazioni. Esistono poi una serie di altri esiti che però prefigurano una messa in sospensione della posizione del giovane, come ad esempio la dicitura «in osservazione», nel caso non infrequente di coscritti che presentavano una forma evidentemente ritenuta leggera o comunque guaribile di una certa patologia. Questi erano inviati presso una struttura ospedaliera dell'esercito per essere sottoposti ad una visita più approfondita, altri erano rimandati ad una visita suppletiva nella stessa sessione di leva, a volte anche solo per accertare se la malattia o la disfunzione riscontrate erano frutto di simulazione.

Da quanto abbiamo visto, dunque, possiamo disporre di più visite riguardo ad una stessa persona, in dipendenza, principalmente, del numero di volte che un giovane era dichiarato rivedibile. A causa di ciò, per un singolo individuo possiamo riscontrare problemi di salute o caratteristiche fisiche difformi tra una visita e l'altra. Per evitare la duplicazione di informazioni riguardanti una stessa persona, non abbiamo preso in considerazione tutte le informazioni raccolte nella documentazione, ma solo quelle relative alla prima visita ${ }^{1}$. Tuttavia, nel caso di una singola visita, potevano essere evidenziate più patologie o disabilità, $\mathrm{o}$ anche essere segnalate problematiche ritenute secondarie. In questi casi abbiamo tenuto presenti tutte le segnalazioni. Per questo stesso motivo, quindi, vi sono anche degli abili ai quali è attribuita una disabilità che, in altri casi, poteva condurre anche all'esonero. Bisogna però dire che la visita di leva non sanciva in via definitiva l'idoneità del giovane al servizio militare. Il coscritto, infatti, era visitato ancora una

Sulla base del fatto che una medesima patologia può aver dato luogo a una dichiarazione di idoneità, piuttosto che di rivedibilità ci dà anche delle indicazioni sul grado di gravità della malattia o della problematica rilevata. 
volta quando giungeva al reparto di destinazione. Solo allora veniva accertato se effettivamente poteva affrontare la vita militare.

L'insieme delle caratteristiche fisiche dei singoli giovani e le cause di inidoneità temporanea o definitiva al servizio militare costituiscono un patrimonio informativo importantissimo nello studio della salute delle popolazioni. A tal proposito, nell'ambito di un progetto che da anni stiamo conducendo sulle fonti militari relativamente alla Provincia di Udine abbiamo raccolto gli esiti di oltre 300.000 visite di leva relativamente a I79 diversi comuni che suddividevano il suo territorio dal punto di vista amministrativo.

In questo lavoro, poiché la copertura delle leve raccolte non è omogenea dal punto di vista temporale in tutto il Friuli, utilizziamo solo una parte di questo patrimonio informativo, quella cioè che si riferisce ai coscritti delle classi 1846-I89o le cui evidenze sono relative in forma pressoché completa per tutto il territorio. Le registrazioni utilizzate risultano pertanto essere I77.260, sulle 339.025 che costituiscono l'intera base di dati.

Gli esiti della nostra ricerca sono riportati in una serie di carte tematiche. La carta impiegata come base di riferimento fa parte di una serie di mappe storiche georeferenziate in cui, a varie date, sono stati ricostruiti i confini amministrativi della Provincia del Friuli. Quella utilizzata in questo lavoro riporta i confini comunali alla data del censimento della popolazione del $\mathrm{I}^{8} 8 \mathrm{I}^{2}$.

\section{Risultati}

Come si è visto, benché la leva fosse universale, non tutti gli aventi obbligo vi si sottoponevano, almeno in patria. Numerosi, infatti, erano i giovani emigrati che erano arruolati spesso senza visita o che non rispondevano alla chiamata, risultando così renitenti. Naturalmente l'emigrazione, che poteva essere temporanea o definitiva, benché diffusa in tutti i comuni del territorio, vi incideva in maniera differenziata. Oltre a ciò è anche necessario considerare che da un anno all'altro la quota di giovani all'estero poteva cambiare notevolmente, in relazione anche del periodo dell'anno in cui si teneva la visita.

Per descrivere sia la diffusione delle principali patologie sul territorio sia la loro evoluzione nel tempo è quindi necessario tenere in considerazione solo la documentazione relativa alle visite effettivamente svolte. Naturalmente le altre informazioni sono anch'esse importanti per studiare altri aspetti, come

2 I criteri di realizzazione delle carte storiche sono descritti nel dettaglio in Fornasin 2005b. 
la distribuzione territoriale delle migrazioni, meno utili per gli obiettivi che ci siamo posti in questo lavoro. D'altro canto, però, si deve pure considerare che gli emigranti erano in genere più sani dei non emigranti, anche perché l'emigrazione era di tipo economico e quindi coinvolgeva le persone che effettivamente erano in grado di lavorare. Per questa ragione, quindi, la salute di alcuni territori può risultare peggiore di quella di altri per la semplice ragione che i flussi migratori vi erano più consistenti e che quindi risultava assente una quota importante della popolazione più sana.

L'interesse delle autorità militari era valutare la capacità dell'individuo a poter vestire la divisa ed essere in grado di svolgere tutte quelle mansioni proprie di un soldato. Tra queste, oltre al maneggio delle armi, era necessaria una certa prestanza fisica, variamente misurata attraverso la statura o l'ampiezza del torace, Oltre all'integrità fisica, il giovane doveva avere uno stato di salute adeguato ${ }^{3}$.

Le esigenze militari si riflettevano inevitabilmente anche sui criteri nosologici adottati nelle visite. Le infermità dei giovani non dovevano essere individuate a scopi di cura, ma solo a scopi pratici: il soldato doveva essere in grado di marciare e maneggiare le armi. Non sempre, quindi, per le malattie è individuabile l'eziologia e assai raramente la patogenesi, obiettivo che ci porremo in successivi lavori, quando studieremo anche l'evoluzione dello stato di salute dei giovani nel tempo. Coerentemente con gli obiettivi della visita, dunque, spesso sono descritti i sintomi e, soprattutto, gli organi coinvolti. Nonostante questi limiti, la documentazione offre l'eccezionale opportunità di dare uno sguardo allo stato di salute di una popolazione del passato non solo attraverso quelle informazioni che solitamente si usano a tal fine: le cause di morte, ma soprattutto indagando malattie o infermità invalidanti ma non mortali, come ad esempio le affezioni della tiroide o lo stato della dentatura.

Nella figura 2 proponiamo la carta relativa alla distribuzione geografica dei giovani che in occasione della loro visita di leva non erano stati giudicati idonei al servizio militare. La carta offre in qualche misura un quadro generale dello stato di salute della popolazione giovane nelle sue diverse articolazioni generali. Non sono state tenute distinte le cause di inidoneità dovute al non raggiungimento dei limiti antropometrici (statura e circonferenza

3 Non doveva nemmeno avere caratteristiche fisiche che potevano risultare troppo sgradevoli in un contesto in cui spesso tante persone erano costrette a convivere in spazi angusti. Anche una eccessiva sudorazione poteva essere quindi causa di inidoneità. 


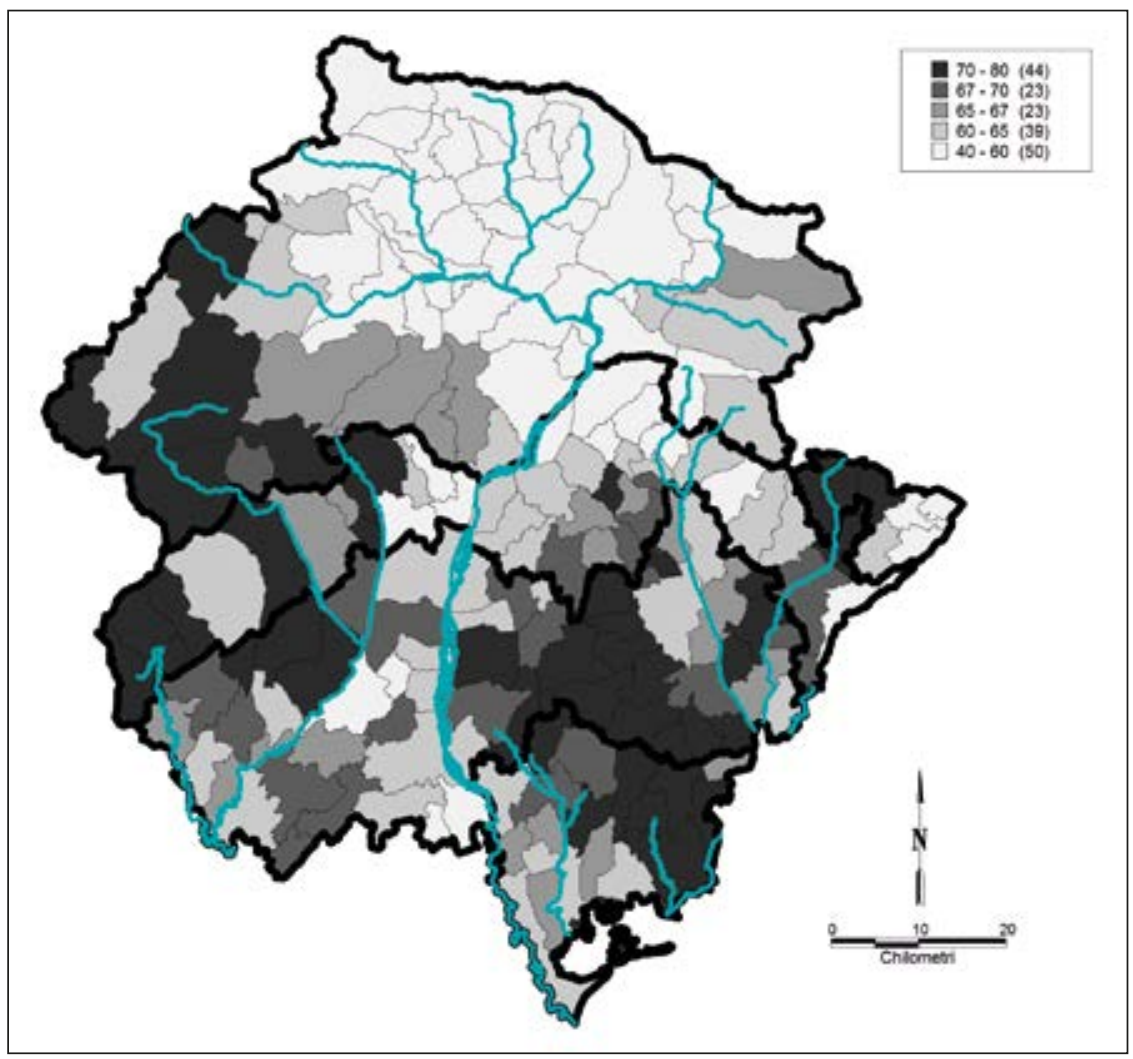

Fig. 2. Distribuzione geografica dei non idonei al servizio militare alla prima visita di leva (\%). Friuli, generazioni I846-I89o.

toracica) da quelle dovute a malattia perché molto spesso la bassa statura o la deficienza toracica avevano origine proprio dallo stato di salute del giovane.

La carta permette di individuare una chiara distribuzione delle zone più e meno favorite in quanto a salute della popolazione maschile. L'area montuosa settentrionale è sicuramente quella più avvantaggiata, in posizione intermedia si trova la parte centrale del Friuli, mentre le aree in cui la percentuale di rivedibili e riformati è maggiore si trovano nella parte orientale e occidentale della provincia.

Questa carta non rappresenta una sorpresa. Sono oramai diversi gli studi che dimostrano come, contrariamente ad una ancora consolidata opinione, le aree montane non fossero aree depresse, come spesso lo sono invece oggi. Quanto già è stato visto sui livelli di alfabetizzazione o sulla struttura economica si osserva, quindi, anche sui livelli generali di salute, che in qualche 
modo dovrebbero essere ad essa collegati. Vi sono però altri elementi che rendono il quadro interpretativo un po' più complesso.

Nella figura 3 , ad esempio, presentiamo la distribuzione territoriale di una patologia assai diffusa in Friuli ancora nel XIX secolo, ovvero l'ipertrofismo tiroideo indicato solitamente nella fonte come "gozzo». Questa malattia è imputabile alla carenza di iodio nell'organismo. La causa è dovuta alla povertà di sali minerali nell'acqua. Uno degli effetti della malattia era un rallentamento dello sviluppo fisico dei più giovani, che si traduceva nella ridotta statura (Tanner 1990, 93). Nei casi più gravi chi era ammalato di gozzo era pure affetto da nanismo. Molto numerosi, quindi, erano gli inidonei al servizio militare a causa della deficiente statura dove maggiore era la diffusione di questa patologia.

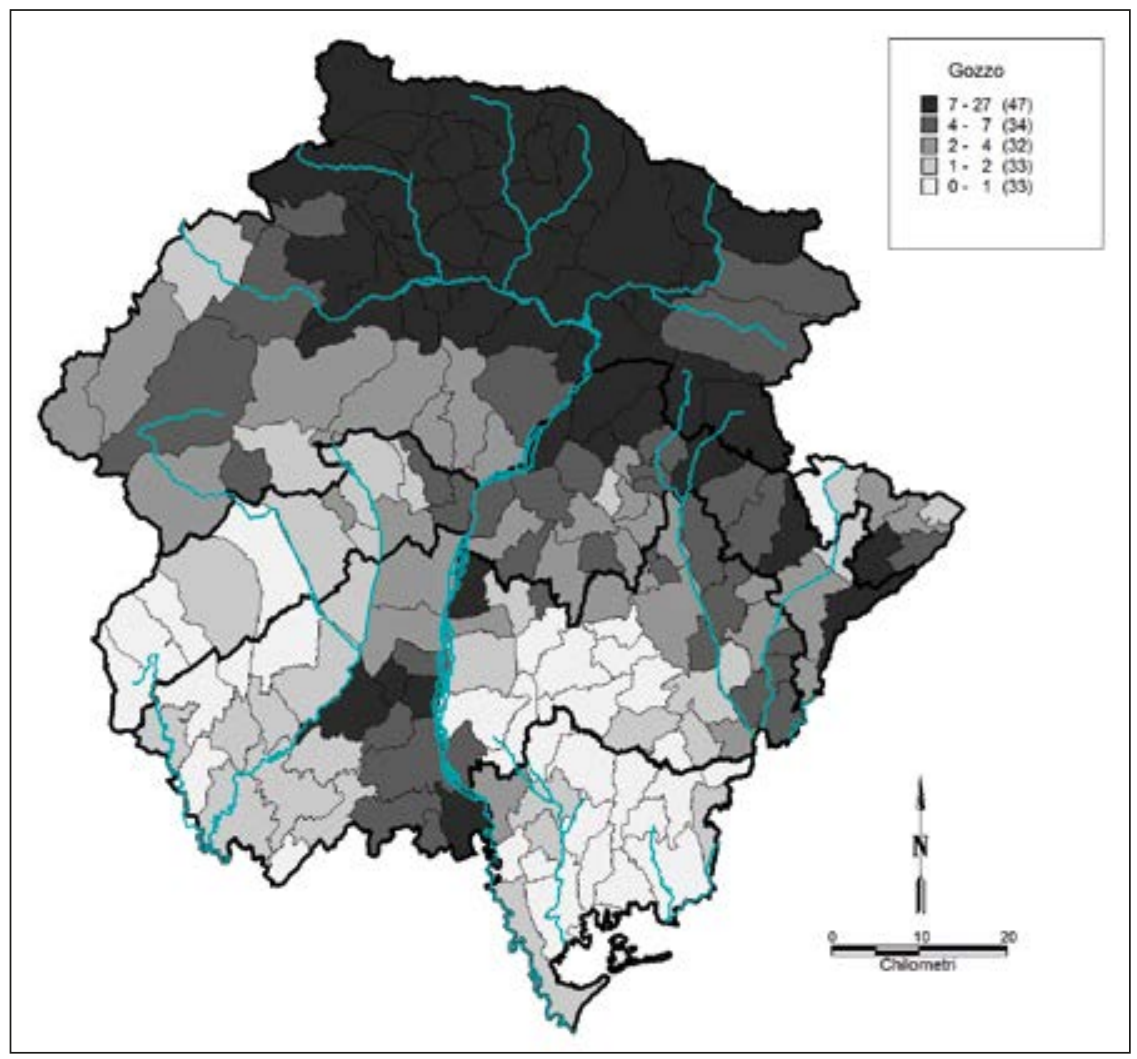

Fig. 3. Distribuzione geografica degli affetti da ipertrofismo tiroideo alla prima visita di leva (\%). Friuli, generazioni 1846-189o. 
Come si può osservare dalla figura, la diffusione del gozzo ricalca in buona misura quanto ci si poteva aspettare. La maggiore concentrazione di coscritti affetti da questa patologia si concentra nelle aree montane settentrionali. Alle più alte quote il contenuto di sali minerali dell'acqua è minore. Anche se la diffusione del gozzo non segue rigidamente la gerarchia delle quote altimetriche, in termini generali, però, la rispetta. La parte di piano, in particolare la pianura umida, è interessata poco o nulla dalla patologia, se non nei comuni che si dispongono lungo l'asse del Tagliamento, il fiume principale che attraversa la provincia. A parte qualche eccezione, il gozzo si configura comunque come una malattia tipica delle alte quote, quindi proprio là dove il numero degli idonei era maggiore.

La figura 4 descrive invece la diffusione di una patologia legata alla funzionalità visiva, ovvero la congiuntivite. Le informazioni contenute nella

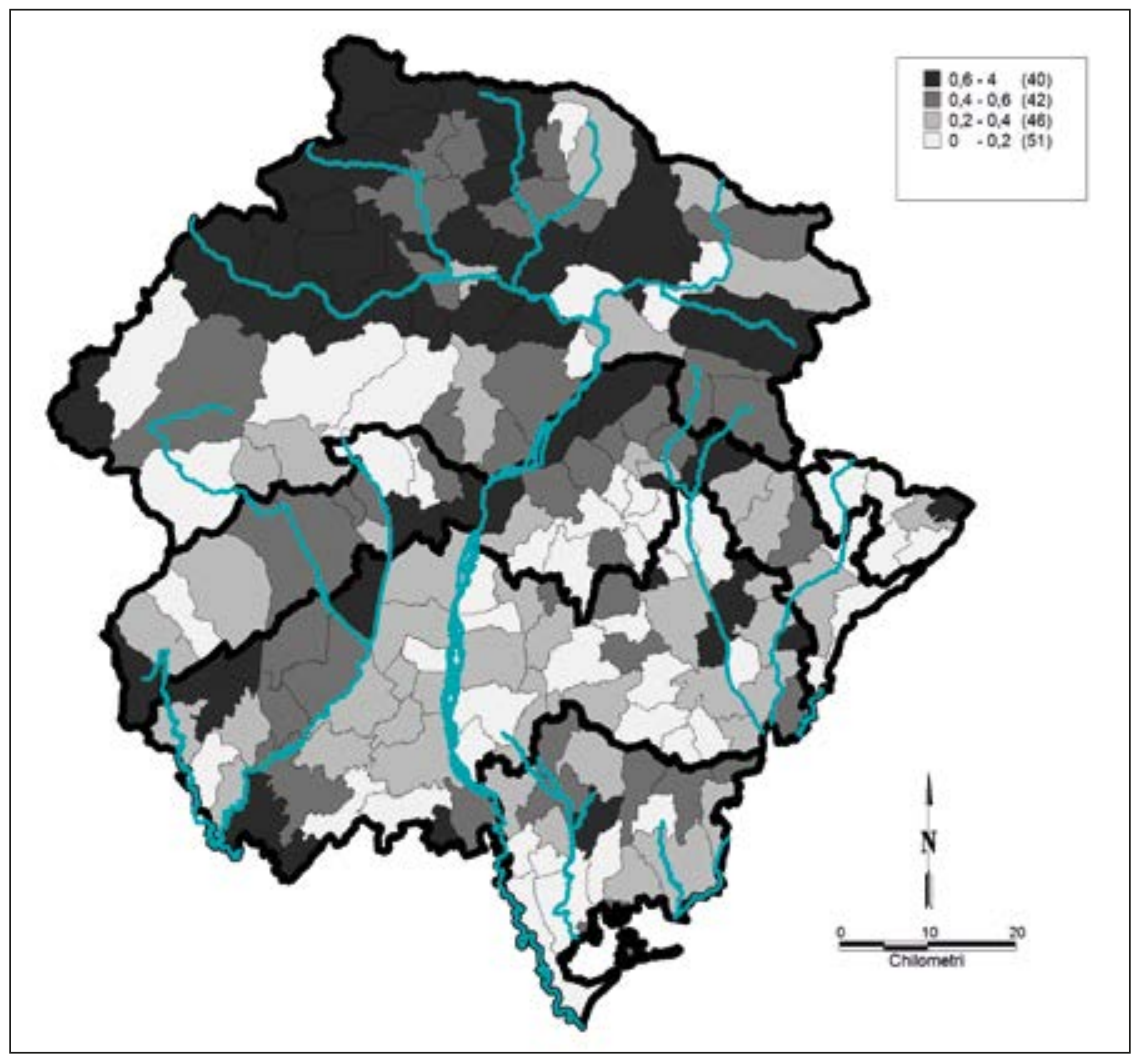

Fig. 4. Distribuzione geografica degli affetti da congiuntivite alla prima visita di leva (\%). Friuli, generazioni I846-189o. 
visita di leva non permettono di determinare la sua causa, che in effetti può essere dovuta a circostanze assai diverse, come infezioni, allergie, irritazioni. La congiuntivite, inoltre, poteva essere il primo stadio del tracoma (Melis, Pozzi 2013). Tuttavia emerge chiaramente una distribuzione territoriale del fenomeno, la cui diffusione è particolarmente evidente nella montagna nord occidentale, mentre è meno diffusa nella zona collinare immediatamente a nord di Udine, nella montagna della Slavia friulana e nella parte più meridionale della pianura umida. Tale diffusione è, forse, da imputarsi anch'essa, almeno in parte, alle caratteristiche delle acque usate per l'igiene personale, ma è probabile che dipenda anche dai rischi per la vista che derivano dalle specifiche occupazioni che si praticavano in territori diversi.

Anche l'ultima carta che presentiamo si riferisce ad un aspetto dello stato di benessere dei coscritti che non può essere automaticamente attribuito ad un'unica causa. Nella figura 5 si può osservare la distribuzione territoriale

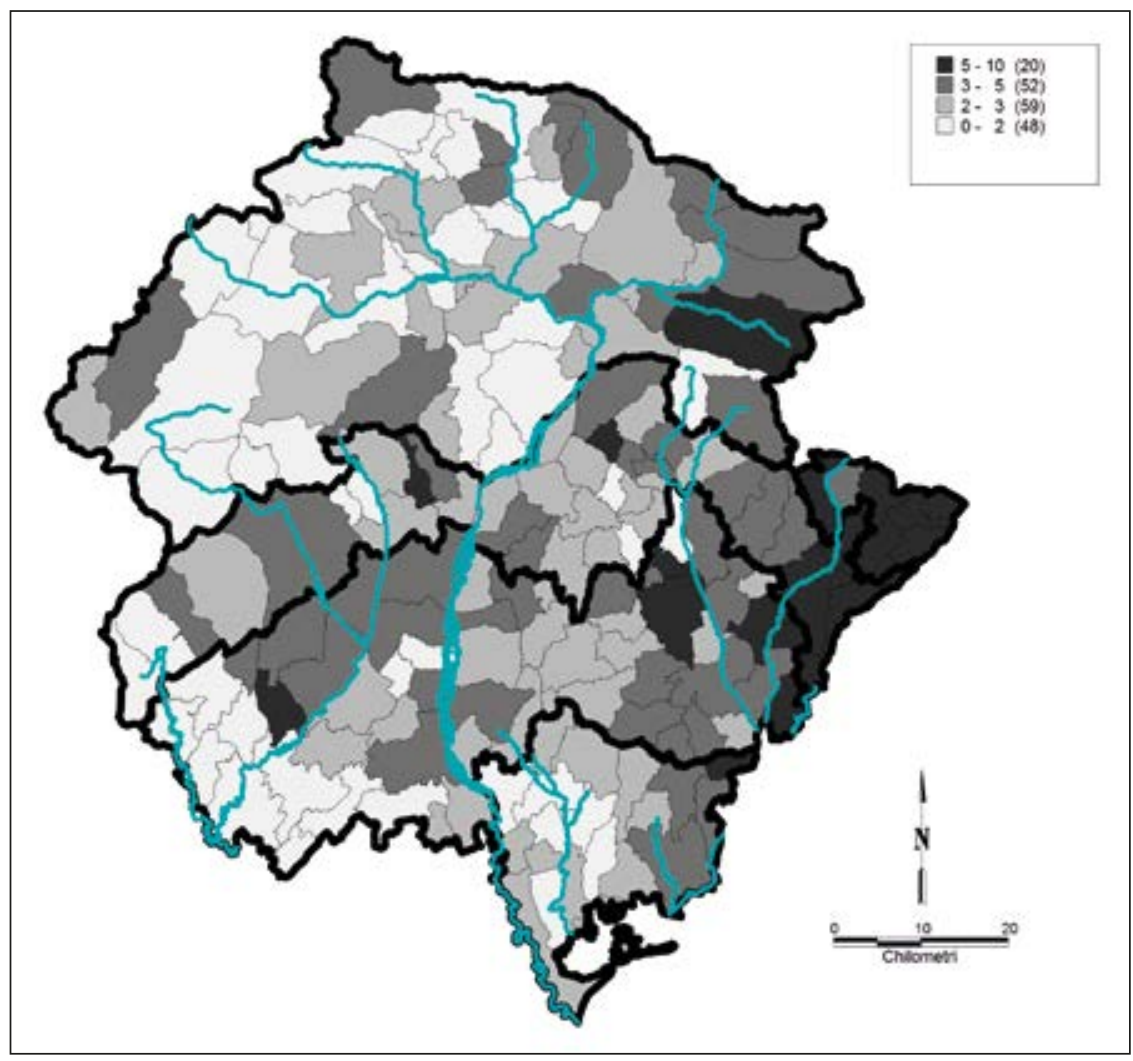

Fig. 5. Distribuzione geografica degli affetti da carie dentale alla prima visita di leva (\%). Friuli, generazioni I846-I89o. 
dei coscritti relativamente ai quali era stato riscontrato un cattivo stato della dentatura.

Come evidenziato dalla carta, anche la distribuzione della carie dentale segue un profilo marcatamente territoriale. I comuni dove essa era maggiormente diffusa sono tutti concentrati nell'estrema parte meridionale del territorio. Procedendo verso est, lungo la zona collinare e la media pianura friulana, la prevalenza della carie tra i giovani visitati diminuisce, ma rimane comunque in quasi tutti i comuni rilevante, tra il $2 \%$ e il 5\%. Queste percentuali sono quasi sempre più basse nella zona meridionale e in quella settentrionale della provincia. Come è noto, la presenza di carie nei denti è dovuta ad una molteplicità di fattori. Tra questi vi sono la predisposizione genetica e il tipo di alimentazione. È possibile che le risultanze che emergono dalla mappa siano da iscriversi ad entrambi. Non è forse casuale, quindi, che quasi tutti i comuni più interessati dal fenomeno siano quelli popolati da minoranze di origine slava, così come siano compresi nelle parti alte della scala alcuni dei centri con caratteristiche più marcatamente urbane della provincia, come Udine, Pordenone e Palmanova, dove si concentrava buona parte degli appartenenti alle classi superiori. In questo caso potrebbero essere quindi i maggiori consumi di zucchero, un bene che poteva essere considerato ancora di lusso nel XIX secolo, a determinare la sua incidenza relativamente più alta nei centri urbani. Restano tutti da chiarire, però, le ragioni per cui bassa pianura e montagna nord occidentale fossero così poco interessate dal fenomeno.

\section{Conclusioni}

Alcune patologie e disabilità sofferte dai giovani ventenni della provincia del Friuli nella seconda metà del XIX secolo mostrano una chiara distribuzione territoriale in relazione a diversi fattori, come l'altitudine o le caratteristiche socio economiche. Naturalmente è importante capire quale sia l'effettiva relazione causale che lega la diffusione di alcune disabilità con queste caratteristiche. La domanda forse più rilevante riguarda la ripartizione dei non idonei al servizio militare che può essere presa come indicatore generale dello stato di salute della popolazione, quella almeno dei ventenni. Come abbiamo visto, la quota più bassa di non idonei al servizio militare si individua nelle aree di montagna, anche se non ovunque con la stessa intensità. Studi recenti hanno ad esempio messo in luce come la montagna fosse relativamente favorita rispetto 
agli altri contesti del Friuli riguardo a diversi fattori socio economici (Fornasin, Lorenzini 20r6), inclusa la qualità dell'alimentazione (Fornasin 2005b).

Non c'è dubbio, però, che in questo territorio erano diffuse delle cause specifiche di disabilità. Abbiamo visto, infatti, che una patologia, il gozzo, era dovuta alle caratteristiche ambientali, ovvero alla bassa salinità dell'acqua usata per l'alimentazione, piuttosto che a fattori socio economici. Un'altra disabilità, ovvero la congiuntivite e, più in generale, le disabilità legate alla vista, erano anch'esse diffuse prevalentemente in montagna, seppure in maniera meno evidente rispetto al gozzo. In questo caso, però, è più difficile individuare una causa certa. Relativamente ad altre patologie non mancano infine le cause di tipo genetico. La mappa che abbiamo presentato riguardo alla diffusione della carie dentale può essere un buon esempio di quanto sostenuto, benché altri fattori possano concorrere a determinare la sua diffusione sul territorio. La carie, infatti, era particolarmente presente dove vivevano e vivono ancora oggi le minoranze di origine slava del Friuli.

Non tutte le patologie le cui mappe sono presentate in questo lavoro possono essere messe in chiara relazione con le cause che le hanno determinate. Troppo poche sono ancora le conoscenze che abbiamo riguardo al passato sulle caratteristiche degli ambienti in cui, concretamente, uomini e donne trascorrevano la maggior parte della loro vita. Del resto si tratta di questioni che ancora oggi sono alla ricerca di una loro compiuta definizione, pur potendo contare sull'esistenza di una quantità di informazioni enormemente maggiore.

Questo lavoro è stato svolto nell'ambito del progetto Friuli/in prin realizzato dall'Archivio di Stato di Udine in collaborazione con il Dipartimento di Scienze Statistiche dell'Università di Udine. 


\section{Bibliografia}

1. G. Alfani 2010, Il Grand Tour dei Cavalieri dell'Apocalisse. L'Italia del «lungo Cinquecento» (1494-1629), Marsilio, Venezia.

2. G. Alvaro 1903 Contribuito alla carta nosografica della Sicilia (malaria, tracoma, vaiuolo), Lugaro, Palermo.

3. J. Bernabeu Mestre 1995, Enfermedad y población. Introducción a los problemas y métodos de la epidemiologia histórica, Seminari d'Estudis sobre la Ciencia, Valencia.

4. M.L. Betri, A. Gigli Marchetti (a cura di) 1982, Salute e classi lavoratrici in Italia dall'unità al fascismo, Angeli, Milano.

5. F. Bianco 1994, Le terre del Friuli. La formazione dei paesaggi agrari in Friuli tra XV e XIX secolo, Astrea-Cierre, Mantova-Caselle di Sommacampagna (VR).

6. M. Breschi, A. Fornasin 2007, La mortalità per genere nei primi cinque anni di vita, Italia 1864-1959, in M. Breschi, L. Pozzi (a cura di), Salute, malattia e sopravvivenza in Italia fra' 800 e'900, Udine, Forum, pp. 243-272.

7. O. Bussini, D. Lanari 2007, Statura e condizioni di salute della popolazione maschile a fine Ottocento: uno studio basato sui registri di leva, in M. Breschi, L. Pozzi (a cura di), Salute, malattia e sopravvivenza in Italia fra '800 e'900, Udine, Forum, pp. 105-133.

8. O. Bussini, D. Lanari, L. Calzola, L. Minelli, Caratteristiche demografiche e socio-sanitarie delle leve militari in Umbria da fine Ottocento a fine Novecento, in C.A. Corsini (a cura di), Statura, salute e migrazioni: le leve militari italiane, Forum, Udine, pp. 47-75.

9. P. Cau, C. Merella, L. Pozzi 2007, Lo stato di salute della popolazione di Alghero fra '800 e '900. Uno studio condotto attraverso i registri militari, in M. Breschi, L. Pozzi (a cura di), Salute, malattia e sopravvivenza in Italia fra' 800 e '900, Udine, Forum, pp. 135-156.

10. G. Di Caporiacco 1967-69, Storia e statistica dell'emigrazione dal Friuli e dalla Carnia, Udine, Edizioni del Friuli Nuovo.

11. G. Donelli, V. Di Carlo 2002, I laboratori della sanità pubblica. L'amministrazione sanitaria italiana tra il 1887 e il 1912, Laterza, Roma-Bari.

12. B. Farolfi 1982, L'antropologia negativa degli italiani: $i$ riformati alla leva dal 1862 al 1886, in M.L. Betri, A. Gigli Marchetti (a cura di) 1982, Salute e classi lavoratrici in Italia dall'unità al fascismo, Angeli, Milano, pp. 165-198.

13. A. Fornasin 1998, Emigrazioni e mestieri in Carnia: la cesura del XIX secolo, «In Alto», pp. 116, 19-40.

14. A. Fornasin 2005a, Patrimonio zootecnico e disponibilità alimentari. Una stima delle calorie di origine animale nel Friuli di fine Settecento, «Rivista di Storia dell'Agricoltura», XLV, 1, pp. 55-84. 
15. A. Fornasin 2005b, I sistemi informativi geografici per la storia del Friuli. Ricerche in corso, «Annales Series Historia et Sociologia», 15, 1, pp. 25-38.

16. A. Fornasin, M. Breschi, M. Manfredini 2011, Houses and individuals in Udine during the cholera outbreak of 1836. A geo-referenced analysis with micro-level data, «Genus», pp. 67, 2, 101-118.

17. A. Fornasin, C. Lorenzini 2016, Integrated Peasant Economy in Friuli $\left(16^{\text {th }}-18^{\text {th }}\right.$ Centuries), in corso di pubblicazione.

18. A.M. Gatti, C.M. Calò, I.S. Piras, Lo stato di salute delle leve militari in Sardegna tra Ottocento e Novecento, in C.A. Corsini (a cura di), Statura, salute e migrazioni: le leve militari italiane, Forum, Udine, pp. 169-191.

19. C. Giovannini 1996, Risanare le città. L'utopia igienista di fine Ottocento, Angeli, Milano.

20. R. Livi 1896, Antropometria Militare, parte 1, Dati antropologici ed etnologici; Atlante della geografia antropologica d'Italia, Giornale medico del Regio Esercito, Roma,

21. M. Livi Bacci 1986, Fertility, nutrition and pellagra: Italy during the vital revolution, "Journal of Interdisciplinary History», XVI, pp. 431-454.

22. Marc, 1883 Censimento della popolazione del Regno d'Italia al 31 dicembre 1881, Roma, Maic.

23. S. Mastroluca, M. Verrascina 2012, L'evoluzione dei contenuti informativi del censimento della popolazione, in «Annali di statistica», pp. 12, 141, 2, 77-119.

24. P.M. Melis, L. Pozzi 2013, Una malattia della povertà: il tracoma infantile in Sardegna, in M. Breschi, L. Pozzi (a cura di), Mortalità e stato di salute dalla nascita alla prima adolescenza: indagini micro in Italia, secoli XIX-XX, Forum, Udine, pp. 157-187.

25. L. Morassi 2002, Il Friuli, una provincia ai margini (1814-1914), in R. Finzi, C. Magris, G. Miccoli (a cura di), Il Friuli-Venezia Giulia, Einaudi, Torino, 1, pp. 5-148.

26. L. Pozzi 2000, La lotta per la vita. Evoluzione e geografia della sopravvivenza in Italia fra' 800 e'900, Forum, Udine.

27. C. Quinzio 1888, Le ernie nei militari, in Annali Universali di medicina e chirurgia, pp. 283, 851, 337-364; 283, 852, 417-443.

28. J.M.D. Snow (1855), On the Mode of Communication of Cholera, John Churchill, London.

29. F.M. Snowden 2006, The conquest of malaria. Italy, 1900-1962, Yale University Press, New Haven.

30. E. Sori 1984, Malattia e demografia, in Franco Della Peruta (ed.), Storia d'Italia. Annali 7. Malattia e medicina, Einaudi, Torino.

31. G. Sormani 1881, Geografia nosologica dell'Italia, "Annali di Statistica» serie 2, vol. 6 . 
32. J.M. Tanner 1990, Foetus into man. Physical growth from conception to maturity, Harvard University Press, Cambridge Ma.

33. E. Tognotti 2000, Il mostro asiatico: storia del colera in Italia, Laterza, Roma-Bari.

34. E. Tognotti 2002, La febbre spagnola in Italia. La storia dell'influenza che fece temere la fine del mondo (1918-1919), Angeli, Milano.

35. L. Torelli 1882, Carta della malaria dell'Italia, Pellas, Firenze. 


\section{SAŽETAK}

Cilj je ovog rada predstaviti zdravstvene mape povijesne populacije. Istraživanja o zdravstvenom stanju populacije u povijesti obično se usredotočuju na uzroke smrti. Naša je namjera predstaviti neke deskriptiune analize o nesmrtonosnim bolestima. Ovaj se rad usredotočuje na provinciju Furlaniju (sjeveroistočna Italija) u drugoj polovici XIX. stoljeća. Korišteni izvori su liste poziva u vojsku. Prikupili smo oko 300 ooo spisa koji se odnose na vojno novačenje između I866. i igo9. (godišta od I846. do I890.). Naš je fokus bio na zdravstvenom stanju dvadesetogodišnjaka $i$ njihove povezanosti sa socioekonomskim i genetskim faktorima, te na utjecaju okoline na njihovo zdravlje. Općenito govoreći, primijetili smo da je sjeverno, planinsko područje što se tiče zdravlja imalo najbolje zdravstvene rezultate, a da su mladići iz istočnih $i$ zapadnih dijelova provincije bili najviše zdravstveno deprivilegirani. Mnoštvo je čimbenika i uzroka odredilo prostornu raspodjelu specifičnih bolesti u provinciji Furlaniji, a mnogi uzroci ostaju nam nepoznati zbog jednostavne deskriptivne analize bolesti nedovoljne da bi ih sve rasvijetlili. Posebno, rasprostranjenost hipertrofije štitnjače gotovo je sigurno uzrokovana kvalitetom vode, a rasprostranjenost zubnog karijesa vjerojatno je povezana s genetskim i prehrambenim faktorima.

Ključne riječi: zdravstvene mape; vojni izvori; Furlanija (Italija); XIX. stoljeće.

\section{RIASSUNTO}

Il presente studio si propone di presentare alcune mappe di salute relative ad una popolazione storica. Gli studi sullo stato di salute delle popolazioni del passato è incentrato solitamente sulle cause di morte. Il nostro obiettivo è di presentare alcune analisi descrittive sulla distribuzione di alcune patologie che non avevano effetto letale. Il lavoro è incentrato sul Friuli (Italia nord-orientale) nella seconda metà dell'Ottocento. La fonte utilizzata è costituita dalle liste di estrazione militare e consiste in circa 300.000 visite di leva svoltesi tra il I866 e il 1909 (generazioni I846-I890). Il nostro principale obiettivo è quello di valutare lo stato di salute della popolazione maschile di 20 anni e la sua associazione con fattori ambientali, socio-economici e genetici. In generale l'area montuosa settentrionale risultava essere la più avvantaggiata, mentre le aree più svantaggiate erano quelle della parte orientale e occidentale della provincia. Molti fattori hanno contribuito a determinare la distribuzione spaziale di alcune malattie specifiche del Friuli, mentre altre restano sconosciute perché le semplici analisi descrittive non sono sufficienti per evidenziarle tutte. In particolare la distribuzione dell'ipertrofismo tiroideo era dovuta quasi sicuramente alla qualità dell'acqua, mentre la distribuzione della carie dentaria era probabilmente legata a fattori di tipo genetico e alimentare.

Parole chiave: Mappe di salute; Fonti militari; Friuli (Italia); XIX secolo. 